\title{
Una década de investigación etnobotánica en la Amazonía legal: ¿qué más se puede hacer?
}

Los grupos tradicionales en la Amazonía poseen formas particulares de ocupación, uso de recursos, generación de servicios y transmisión de conocimientos de valor sustancial para su supervivencia. El objetivo de este trabajo fue analizar los escenarios, desafíos y perspectivas de los estudios etnobotánicos en la Amazonía Legal a través de bibliografías y experiencias del Grupo de Investigación 'Estudios Interdisciplinarios en Botánica' (GPEIB), de la Universidad Estatal de Pará, en un intento de reflexionar sobre los diferentes enfoques propuestos en estas singularidades culturales. Las 63 bibliografías consultadas abarcan los años 2008 a 2018 , siendo obtenidas con 5 palabras-clave asociadas al término Amazonía en las plataformas SciELO, ScienceDirect y Google Scholar. Las experiencias del GPEIB contribuyeron con información relacionada al tema en el período de 2010 a 2018, desarrolladas en seis municipios de Pará. Se observó que las plantas desempeñan funciones y valores en la vida social de las comunidades. Los datos de la investigación mostraron incipientes esfuerzos intelectuales para aproximarse a la colectividad y generar apoyo pedagógico, jurídico y cultural, que pueda ser aplicado como dispositivos legales, formales e institucionalizados. Se carece de diagnósticos participativos para cada grupo investigado, que estén enfocados en la elaboración de mapeos sociales que auxilien a estos grupos a incrementar sus sistemas productivos y a combatir la explotación de sus territorios y la violación de sus formas de vida.

Palabras-clave: Escenarios socioculturales; Etnoconocimiento; Bosque tropical; Instrumentos de gestión; Plantas útiles.

\section{Uma década de pesquisas etnobotânicas na Amazônia legal: o que mais pode ser feito?}

\begin{abstract}
Os grupos tradicionais da Amazônia possuem formas particulares de ocupação, uso de recursos, geração de serviços e transmissão de conhecimentos de grande valor para sua sobrevivência. O objetivo deste trabalho foi analisar os cenários, desafios e perspectivas dos estudos etnobotânicos na Amazônia Legal por meio de bibliografias e experiências do Grupo de Pesquisa 'Estudos Interdisciplinares em Botânica' (GPEIB), da Universidade Estadual do Pará, na tentativa de refletir sobre as diferentes abordagens propostas nessas singularidades culturais. As 63 bibliografias consultadas abrangem os anos de 2008 a 2018 , sendo obtidas com 5 palavras-chave associadas ao termo Amazon nas plataformas SciELO, ScienceDirect e Google Scholar. As experiências do GPEIB contribuíram com informações relacionadas ao tema no período de 2010 a 2018, desenvolvidas em seis municípios paraenses. Observou-se que as plantas desempenham funções e valores na vida social das comunidades. Os dados da pesquisa mostraram esforços intelectuais incipientes para se aproximar da comunidade e gerar respaldo pedagógico, jurídico e cultural, que pode ser aplicado como dispositivos legais, formais e institucionalizados. Faltam diagnósticos participativos para cada grupo investigado, voltados para a elaboração de mapeamentos sociais que ajudem esses grupos a aumentar seus sistemas produtivos e a combater a exploração de seus territórios e a violação de seus modos de vida.
\end{abstract}

Palavras-chave: Cenários socioculturais; Etnoconhecimento; Floresta tropical; Instrumentos de gestão; Plantas úteis.

Topic: Uso Sustentável da Biodiversidade

Reviewed anonymously in the process of blind peer.
Received: 01/02/2021

Approved: 25/02/2021
Flávia Cristina Araújo Lucas (i)

Universidade do Estado do Pará, Brasil

http://lattes.cnpq.br/4286675736752972

http://orcid.org/0000-0002-0752-7206

copaldoc@yahoo.com.br

Yuri Cavaleiro de Macêdo Coelho (iD)

Universidade do Estado do Pará, Brasil

http://lattes.cnpq.br/9256304047716406

http://orcid.org/0000-0001-5175-2104

yuricoelhos15@hotmail.com

Seidel Ferreira dos Santos (iD

Universidade do Estado do Pará, Brasil

http://lattes.cnpq.br/6531723156764196

http://orcid.org/0000-0001-6049-1188

seidelsantos@uepa.br

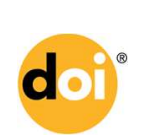

DOI: 10.6008/CBPC2179-6858.2021.002.0021

\author{
Maria Antonia Ferreira Gois (D) \\ Universidade do Estado do Pará, Brasil \\ http://lattes.cnpq.br/7977800987189031 \\ http://orcid.org/0000-0002-5663-7188 \\ mariah.botanica@gmail.com \\ Victor Miranda Leão (D) \\ Universidade do Estado do Pará, Brasil \\ http://lattes.cnpq.br/0437536537187520 \\ http://orcid.org/0000-0002-4696-250X \\ victor_mirandaleao@yahoo.com.br
}

Referencing this:

LUCAS, F. C. A.; COELHO, Y. C. M.; SANTOS, S. F.; GOIS, M. A. F.; LEÃO, V. M.. Una década de investigación etnobotánica en la Amazonía legal: ¿qué más se puede hacer?. Revista Ibero Americana de Ciências Ambientais, v.12, n.2, p.216-232, 2021. DOI:

http://doi.org/10.6008/CBPC2179-6858.2021.002.0021 


\section{INTRODUCCIÓN}

Los pueblos y comunidades tradicionales en la Amazonía son grupos culturalmente diferenciados, representados por pobladores ribereños, agricultores familiares, pueblos indígenas, extractivistas, extractores de caucho, extractores de andiroba, recolectores, herbolarios, curanderos, parteras, quilombolas y otros, que poseen formas particulares de organización, ocupación del territorio, uso de los recursos naturales y generación de servicios. Transmiten conocimientos de un valor sustancial para la percepción, diseño y conceptualización de los recursos, paisajes y ecosistemas, los cuales se convierten en componentes decisivos para la implementación de estrategias de supervivencia (TOLEDO et al., 2010).

Estos conocimientos tradicionales que sedimentan formas de vida en la Amazonía son el resultado de construcciones históricas y, también, de una relación simbiótica con el medio vivido, de tal manera, que la forma de sembrar, trabajar, religión, orden social, ocio, alimentación, medicinas, vestimenta, educación, gobernabilidad y otras expresiones socioculturales provienen de su estrecha relación con la naturaleza (WAWZYNIAK, 2003). Se puede decir que el hombre amazónico es resultado de intercambios históricos entre diferentes pueblos y etnias, lo que posibilitó una herencia revelada en diversas manifestaciones expresadas en la vida cotidiana (LIRA et al., 2016). Por ello, información valiosa contenida en sus cosmologías se revelan, arraigadas en conceptos locales y autosustentables en la búsqueda del buen vivir (MAUÉS, 2012). Además, los pueblos y comunidades tradicionales aportan prácticas conservacionistas que promueven la construcción de una educación ambiental, no solo a través de la mera transmisión de información, sino en la concientización hacia la construcción de valores y hábitos para resolver problemas ambientales (VIEIRA et al., 2018).

Las influencias migratorias dentro de las comunidades tradicionales posibilitan el establecimiento de nuevos grupos sociales, incorporando un bagaje cultural externo en el uso de los recursos regionales, provocando la introducción y/o maximización de variedades exóticas en las costumbres locales (SEMEDO et al., 2007). Al abordar las representaciones de identidades plurales en determinados grupos sociales, la Política Nacional para el Desarrollo Sostenible de Pueblos y Comunidades Tradicionales (BRASIL, 2007) señaló en sus lineamientos que las "mezclas culturales" son condiciones para la expresión de nuevas cartografías sociales, que incorporan religiosidad, ancestralidad, economía, recursos biológicos y la producción de un conjunto de conocimientos resignificados en una comunidad determinada. Además, la formación de la cultura rural brasileña, basada en matrices étnicas originarias de indígenas, negros y blancos, modificó los saberes autóctonos con otros conocimientos y prácticas (COSTA, 2006), dando como resultado peculiaridades regionales, que se manifiestan en las esferas cotidianas como consecuencia de una adaptabilidad temporal.

La importancia de la información etnobotánica para el hombre radica en el conocimiento de datos populares que pueden estar, hasta entonces, restringidos a determinadas personas o regiones (MARTINS et al., 2005), o inclusive, contribuir al mantenimiento tanto de la calidad de vida como de la identificación de los grupos culturales que persisten en determinados lugares, permitiendo la continuidad de la dinámica de 
elaboración y reelaboración del conocimiento etnobotánico (GANDOLFO et al., 2011).

De la biodiversidad amazónica, muchas publicaciones registran innumerables especies de plantas utilizadas por las poblaciones tradicionales, la mayoría de las cuales son desconocidas para la ciencia y las sociedades urbanas no tradicionales. Un mundo nuevo que se mide por su inconmensurabilidad, de donde emerge el aspecto ambiental, biológico y sociocultural. Por otro lado, Val (2014) enfatizó que esta diversidad necesita aprender a enfrentar los desafíos de origen antrópico que avanzan en la contemporaneidad. Algunos de estos desafíos se originan localmente y producen efectos que interfieren en diferentes procesos, como en la reducción de áreas donde se encuentran diversas especies, aumento de la explotación indiscriminada de plantas y degradación ambiental, además de posibilitar la desaparición de saberes dada la modernización de los sistemas culturales. Así, los estudios etnobotánicos han contribuido a comprender la interacción entre los pueblos y los recursos botánicos, aportando elementos sobre el perfil sostenible del medio ambiente (SILVA et al., 2015).

En este contexto, el objetivo de este trabajo fue analizar los escenarios, desafíos y perspectivas de los estudios etnobotánicos en la Amazonía Legal a través de bibliografías y experiencias del Grupo de Investigación "Estudios Interdisciplinarios en Botánica" (GPEIB), de la Universidad Estatal de Pará, en un intento de reflexionar sobre los diferentes enfoques propuestos en estas singularidades culturales, con el fin de contribuir a la inclusión social de los pueblos tradicionales y valorar sus tradiciones y culturas.

\section{MATERIALES Y MÉTODOS}

El presente trabajo se elaboró a través de un levantamiento bibliográfico de archivos publicados entre 2008-2018 sobre el tema, y a partir de las experiencias de los autores en el GPEIB, entre los años 20102018, en comunidades, ferias y mercados de las ciudades de Belém (centro y distrito de Mosqueiro), Capanema, Abaetetuba (centro e islas), Altamira (ribereños) y Vigia. Se realizaron búsquedas bibliográficas con las siguientes palabras-clave, en portugués e inglés: (I) etnobiología; (II) etnobotánica; (III) etnomedicina; (IV) sociobiodiversidad; (V) conocimiento tradicional; y (VI) plantas útiles, todas combinadas con la palabraclave 'Amazonía'. Las herramientas de búsqueda utilizadas fueron: Google Scholar', hasta la tercera página de búsqueda; ScienceDirect ${ }^{2}$, hasta la segunda página de búsqueda; y SciELO ${ }^{3}$, hasta la última página de búsqueda. No se activaron filtros de búsqueda en las plataformas y se adoptó como criterio básico para este procedimiento el grado de importancia de la publicación.

Fueron descartados artículos que no reflejaban la realidad del conocimiento etnobotánico en la Amazonía Legal y privilegiados aquellos con levantamientos completos, es decir, que contemplaran desde el perfil de la comunidad/municipio/feria/mercado hasta el listado de las especies vegetales encontradas en estas localidades; recolección de muestras botánicas, etnoconocimientos asociados a las plantas, así como sus formas de uso, manipulación y/o conservación. Producciones científicas del GPEIB fueron incluidas en

\footnotetext{
${ }^{1}$ http://scholar.google.com.br

2 http://www.sciencedirect.com

3 www.scielo.org/
} 
este trabajo (GERMANO et al., 2014; LOBATO et al., 2014; 2016; 2017; CARMO et al., 2015; MOURA et al., 2016; GOIS et al., 2016; ALVES et al., 2017), considerando el cumplimiento de los criterios anteriormente establecidos.

El levantamiento final incluye artículos científicos nacionales e internacionales, capítulos de libros y obras completas, trabajos de tesis y artículos de sitios web. En total, este trabajo recogió 79 archivos, de los cuales solo 63 fueron analizados por cumplir plenamente con los criterios establecidos.

\section{RESULTADOS Y DISCUSIÓN}

La influencia del conocimiento local de las comunidades en el uso, manejo y conservación de la diversidad vegetal

Los conocimientos adquiridos en la Amazonía denotan que las plantas constituyen parte de la vida social de las poblaciones, desempeñando funciones y valores en su medio, sean materiales o simbólicos. Las especies botánicas de esta región son cultivadas para fines alimentarios, terapéuticos, artesanales, madereros, de construcción, entre otros. La prevalencia de una o más categorías se producirá en función de la ubicación geográfica, los problemas que experimenta la población, la estima por este recurso, el espacio disponible para el cultivo y los aspectos económicos y socioculturales.

Los temas más discutidos en este estudio se basan en el levantamiento realizado por Oliveira et al. (2009), quienes constataron que la mayor parte de la investigación en etnobiología trata exclusivamente sobre plantas medicinales; conocimiento, uso y manejo de recursos vegetales; origen y flujo de conocimientos; etnotaxonomía; y pérdida de conocimientos.

\section{Etnobotánica de plantas medicinales y para uso ritualista y la etnofarmacología}

La propagación y uso de especies medicinales es una práctica muy común entre los pueblos amazónicos, tanto que la mayoría de los artículos levantados se refieren a plantas medicinales, ya que estas, por sus propiedades fitoquímicas y fitoterapéuticas, han despertado gran interés por las potencialidades económicas que representan. Familias botánicas como Lamiaceae, Fabaceae, Asteraceae y Euphorbiaceae frecuentemente representan la mayor riqueza de especies, según levantamientos en comunidades amazónicas (SANTOS et al., 2008; SANTOS et al., 2014; VEIGA et al., 2015; ALVES et al., 2017). Para Lamiaceae y Asteraceae, las aplicaciones terapéuticas se extienden más allá de las zonas tropicales, siendo también numerosas en América del Norte y Europa. El uso abundante de plantas de estas familias, tanto por poblaciones de regiones templadas del hemisferio norte, como por habitantes de regiones tropicales del hemisferio sur, probablemente esté relacionado con el tránsito de recursos biológicos realizado antes y durante la colonización del país. Estos intercambios facilitaron la incorporación de innumerables especies de otras áreas del globo en la cultura local/regional, consiguiendo su aclimatación y naturalización. Para Fabaceae y Euphorbiacea, Das et al. (2015) demostraron que la abundancia de estas familias en los estudios etnobotánicos se justifica en la diversidad de usos y aplicaciones de sus diferentes especies por los pueblos tradicionales. 
En los estudios de Vasquez et al. (2014), en tres comunidades ribereñas, las enfermedades más frecuentes fueron tratadas con 171 especies, pertenecientes a 65 familias, entre las cuales fueron más citadas la menta (Mentha arvensis L.), la ruda (Ruta graveolens L.) y la naranja (Citrus sinensis Osbeck), siendo la decocción de las hojas el procedimiento más común para el tratamiento de enfermedades relacionadas a los sistemas digestivo y respiratorio. Algunas especies destacan repetidamente en artículos científicos por exhibir altos niveles de concordancia en su uso (SANTOS et al., 2008; CARNEIRO et al., 2010; VEIGA et al., 2015; ALVES et al., 2017): oreganón (Plectranthus amboinicus (Lour .) Spreng.); boldo brasileiro (Plectranthus barbatus Andrews); figatil (Vernonia condensata Baker); paico (Dysphania ambrosioides (L.) Mosyakin \& Clemants); C. sinensis Osbeck; M. arvensis L.; cidraero (Lippia thymoides Mart. \& Schauer y Lippia alba (Mill.) N.E.Br.); bellaco caspi (Himatanthus sucuuba (Spruce ex Müll.Arg.) Woodson); hierba luisa (Cymbopogon citratus Stapf); copaiba (Copaifera multijuga Hayne); andiroba (Carapa guianensis Aubl.); R. graveolens L. y coirama (Kalanchoe brasiliensis Cambess). La creciente demanda por el consumo de estas plantas responde a emergencias sanitarias, su facilidad de acceso, bajo costo y compatibilidad con las tradiciones populares.

La curación de enfermedades también es posible cuando hay un proceso de resignificación de las plantas medicinales, que pasan a adquirir poderes religiosos, influenciadas por diferentes orígenes y etnias, especialmente indígenas y/o prácticas religiosas afrobrasileñas. En los relatos de Albuquerque et al. (2012), las prácticas curativas en la ciudad de Soure, en la isla de Marajó, Pará, se expresaron a través de la verbalización de una interlocutora, al afirmar que antes de los médicos y científicos, los responsables por la salud de las personas eran los curanderos y parteras. En otro relato, una curandera anuncia que con el auxilio de los seres que la guían, puede curar tanto enfermedades espirituales (alteración de espíritus, por ejemplo) como aquellas de causa física ('tocedura', 'quebradura'), aunque en casos más urgentes recomienda buscar atención médica o especializada. Este dato converge con lo enfatizado por Cassino (2010), quien interpretó las enfermedades en una conceptualización cultural y simbólica de curación, siendo acompañada por rezadores especialistas que realizan tales actividades con el fin de contribuir a la promoción de la salud en la comunidad.

En las comunidades ribereñas amazónicas, la diarrea infantil, por ejemplo, puede ser causada por fenómenos naturales o sobrenaturales y, en este contexto, aspectos culturales, con simbologías y tabúes involucrados en su concepción, así como reglas de comportamiento, se relacionan con su prevención. Cassino (2010) informó que el tratamiento comúnmente es realizado con baños aromáticos, preparados con las hierbas: Anamú (Petiveria alliacea L.); ruda (R. graveolens L.); menta (Mentha x piperita L., pro spec. \& Hylander); catinga de mulata (Aeollanthus suaveolens Mart. ex Spreng.); y piñón negro (Jatropha gossypiifolia L.). En este mismo escenario, el autor también constató que la decocción de las hojas del curibano (Justicia pectoralis Jacq.) y de la salva-do-marajó (Hyptis crenata Pohl) sirven como incienso para sahumar; el baño realizado con el macerado de la hoja de Tapekue (Acanthospermum Schrank) elimina las molestias; y la decocción de la hoja de isula huayo (Siparuna Aubl.) limpia el cuerpo.

Los resultados de las publicaciones sobre plantas medicinales han provocado en la sociedad fuertes influencias positivas sobre la valorización del conocimiento médico-popular, que no está aislado de la 
investigación realizada en las academias científicas. Por el contrario, han propiciado el descubrimiento de numerosos fármacos, legitimados por el uso de las poblaciones durante un largo período y en procesos transgeneracionales vinculados a fuertes tradiciones de sus usuarios. La mayoría de estos usos notificados tenían el conocimiento tradicional como base de su efectividad, dosis, efectos adversos y reproducibilidad. Así, la información captada en los trabajos de campo junto a los conocedores de las tradiciones ancestrales sobre el uso de la biodiversidad se tornó fundamental para la formulación de nuevos medicamentos y la cura de enfermedades.

El Consejo Colegiado de la Agencia Nacional de Vigilancia Sanitaria (ANVISA) aprobó dos reglamentos que pueden ayudar a la población a acceder a los productos elaborados con plantas de la biodiversidad brasileña ${ }^{4}$. ANVISA actualizó el registro de medicamentos fitoterapéuticos y creó una notificación para los Productos Tradicionales Fitoterapéuticos. La Resolución RDC 26/2014, publicada en el Diario Oficial de la Federación (DOU), en mayo de 2014, definió como primera norma: los Productos Tradicionales Fitoterapéuticos serán autorizados mediante la demostración de uso seguro en humanos por un período prolongado, determinado por la Agencia, de mínimo 30 años; segunda norma: presentación de una lista de 43 plantas de uso común que podrán producir medicamentos sin necesidad de comprobación adicional de eficacia y seguridad. Este nuevo registro simplificado comprende especies brasileñas y plantas reconocidas, así como especies de registro simplificado, reconocidas como seguras en otros países como Canadá y la Unión Europea.

Por lo tanto, a partir de levantamientos etnofarmacológicos, documentación tecnocientífica y estudios toxicológicos pre clínicos y clínicos, se promueve la construcción de importantes marcos normativos para el sector de la fitoterapia en el país. A pesar de los caminos victoriosos ya recorridos, Vendruscolo et al. (2010) lamentaron la falta de estudios químicos y biológicos de muchas especies encontradas en los levantamientos; muy pocas plantas medicinales tienen usos comprobados en la literatura especializada.

El fortalecimiento de la etnofarmacología fue evaluado por Val (2014) como esencial para el avance científico y en los procesos de inclusión social y generación de ingresos. Las investigaciones en bioprospección, relacionadas con el descubrimiento de nuevas moléculas, han avanzado bastante en la Amazonía debido a las actividades de investigación que realizan varios grupos de "Química de Productos Naturales" vinculados a cursos de posgrado e iniciación científica en los diferentes Estados de la Amazonía Legal (ASTOLFI FILHO et al., 2015). Sin embargo, el escenario actual sobre este tema dentro de las comunidades es muy similar al de miles de años atrás. Los pueblos de la Amazonía continúan tratando sus enfermedades mediante brebajes, elaborados a partir de sus conocimientos tradicionales, conteniendo, muy probablemente, principios activos esenciales para el desarrollo de nuevos medicamentos y sin acceso a medicamentos modernos y avances científicos. 


\section{El valor sociocultural, formas de uso y manejo de plantas útiles}

En cuanto a las plantas alimenticias y su consumo sostenible, el valor nutricional y sociocultural de frutas, verduras, legumbres y hortalizas promueve la calidad de vida de los pobladores. La diversidad de ambientes agrícolas y forestales (patio trasero, huerto y bosque) da como resultado una abundancia de recursos naturales, siendo los patios traseros alrededor de la casa un espacio de gran diversidad de alimentos que satisfacen la demanda familiar, conservando las hierbas en lechos o canteros suspendidos. En las áreas de huerta se coleccionan especies experimentadas y manejadas en intercambios con parientes y vecinos, con una amplia representatividad de yuca (Manihot esculenta Crantz), maíz (Zea mays L.), sandía (Citrullus vulgaris Schrad.), zapallo (Cucurbita moschata Duchesne), Mashishi (Cucumis anguria L.), ñame (Dioscorea spp.) y frijol (Vigna sp.). Es muy común encontrar un gran número de etnovariedades en muchos de estos cultivos agrícolas.

En patios traseros urbanos de Abaetetuba, Lobato et al. (2016) encontraron fuentes de proteínas $(0.0036 \mathrm{~kg})$ y carbohidratos $(0.0544 \mathrm{~kg})$, con los niveles más altos provenientes del asaí (Euterpe oleracea Mart.); y lípidos presentes en el coco (Cocos nucifera L.) y en el aguacate (Persea americana Mill.), 0.03349 kg y 0.01006 kg, respectivamente. La acerola (Malpighia punicifolia L.); guayaba (Psidium guajava L.); papaya (Carica papaya L.) y naranja (C. sinensis Osbeck) son ricas en vitamina C. El asaí siempre ha estado presente en los registros de estudio, con especial relevancia por sus múltiples usos, ya sean alimentarios, medicinales, para pequeñas construcciones rurales, o por su uso en utensilios y herramientas domésticas y de pesca.

El valor sociocultural y de subsistencia que tienen ciertas plantas en una comunidad fue evaluado por Germano et al. (2014), en dos comunidades ribereñas, en el estado de Pará, en relación a las palmeras, donde se identificaron 22 especies, para ocho categorías de uso, siendo las de mayor importancia la anjá (Attalea maripa Mart.), Asaí (E. oleracea Mart.), palma de cabecinegro (Manicaria saccifera Gaertn.), Aguaje (Mauritia flexuosa Lf) y jupati (Raphia taedigera Mart.), que obtuvieron los mayores índices de diversidad en su uso. Frecuentemente, el asaí aparece como una de las más valiosas para los residentes, lo que confirma la hipótesis de que el uso de las palmeras está influenciado principalmente por la importancia social, cultural y económica que representa una especie para los residentes.

Las formas de cultivo y técnicas de manejo del uso de recursos se manifiestan como una fuerte influencia de las tradiciones indígenas. El uso de técnicas tradicionales desarrolladas con herramientas artesanales sigue siendo una realidad tecnológica en la agricultura familiar amazónica. Legados autóctonos, que incluyen el trabajo familiar, predominan en la selección y el mejoramiento genético empírico, sobre todo, de forma precisa y altamente selectiva. Robert et al. (2012) consideraron la complejidad de estos procesos prioritarios para el autoconsumo de las poblaciones, ejemplo de ello es el cultivo de la yuca amarga, que para su transformación de una raíz venenosa en una harina "especial" -con sabor, consistencia, color y aroma apreciados en hogares y mercados- requiere un conjunto extremadamente elaborado de gestos, técnicas, instrumentos y conocimientos. 


\section{'Desarrollo' económico, realidad ambiental y comunidades tradicionales}

Los debates que tratan sobre la expropiación de los ambientes naturales amazónicos, justificados por la ideología económica del progreso, el aumento de la productividad, además de la publicidad estereotipada de sostenibilidad, a menudo han sido secundados como cuestiones ambientales fundamentales. La visión del progreso a través de las innovaciones tecnológicas, según Moran (2010), desconsidera la complejidad y el carácter dinámico y adaptativo de los seres vivos que conforman los ecosistemas, además de conducir a la dilapidación del patrimonio genético de la biodiversidad. Estos efectos negativos se integran en una concepción de la naturaleza como algo estático, pasivo de ser dominado y aprovechado en favor de intereses particulares (OLIVEIRA et al., 2011).

En las experiencias del GPEIB, se encontró que las comunidades, tanto las más distantes y aisladas de las ciudades, como las más cercanas, han mantenido sus repertorios tradicionales, incorporando nuevos elementos, en un tránsito de influencias bioculturales; continúan preservando el conocimiento construido sobre la relación entre el hombre y la naturaleza, como evidencian Santos et al. (2012). El uso de los términos "erosión", "pérdida" de conocimientos o "proceso de urbanización" y su influencia en la reproducción de sus formas de vida, necesita ser reconceptualizado y no radicalizado ni generalizados. Es importante considerar que los descendientes de antiguas poblaciones tradicionales mantienen la memoria de los tiempos pre urbanos, dando continuidad a ciertos rasgos culturales que persisten, incluso en medio de la nueva realidad heterogénea propia de las áreas urbanas (GANDOLFO et al., 2011). Esta discusión considera que, a pesar de la presión ejercida a favor del "avance tecnológico", que desconsidera las memorias bioculturales convirtiéndolas en componentes superfluos y desechables, y desperdiciándolas como fuente de sabiduría e inspiración-, estas aún sobreviven y perpetúan conocimientos sui generis que interconectan el pasado, el presente y el futuro de la sociobiodiversidad. Frente a estos patrones que se mueven globalmente, este punto debe ser fuertemente considerado en las investigaciones etnobiológicas.

Las experiencias de organización comunitaria y empoderamiento de postura crítica han crecido en toda la Amazonía. La movilización del conocimiento científico, involucrando a especialistas (científicos), gestores y personas de la sociedad civil, ya se ha convertido en una realidad, aunque todavía se da de manera efímera. Para el Archipiélago de Marajó, la Nueva Cartografía de la Amazonía (BOLETIM INFORMATIVO, 2014) trajo una amplia discusión sobre problemas territoriales, transformaciones socioambientales y estrategias de acción en nueve municipios del archipiélago de Marajó, porción insular del estado de Pará, durante el periodo 2003 a 2014. Se debatieron narrativas sobre la devastación acelerada de los recursos madereros, la privatización de los asaís y la expansión del monocultivo emprendido por la agroindustria.

La integración científica y cultural de los saberes en la Amazonía, resultado del intercambio entre investigadores y residentes locales, es de suma importancia para romper las fronteras de la percepción local de la validez de los saberes culturales. De esta forma, se favorece la mejora de las políticas públicas orientadas a programas de salud popular, recuperación ambiental, uso sostenible de los recursos naturales, entre otros, mediante la democratización del conocimiento a través de la divulgación científica. En caso 
contrario, como afirma Heraldo Maués,

[...] seguiremos teniendo propuestas y acciones de salud ajenas a la realidad social y entendidas a partir de conceptos cosmológicos o visiones del mundo completamente disociados de las cosmologías y del ethos de las poblaciones rurales de la Amazonía - para quedarnos solo en ellas - lo que constituye un serio obstáculo para su eficacia y efectividad. No creo, sin embargo, que sea exagerado remitir también al lector -en esta conclusión- al artículo de Buchillet (2000) que aborda las acciones de salud en el tratamiento de la tuberculosis entre las poblaciones indígenas brasileñas, donde estos temas están fuertemente retratados. (MAUÉS, 2012)

De esta forma, se conjetura que el conocimiento tradicional, a pesar de sufrir alteraciones a lo largo del tiempo, es transgeneracional, debido a la inserción de nuevos actores sociales o transformaciones en las formas de vida en el territorio. Así, en una comunidad los saberes existirán, aunque estén vinculados a nuevas percepciones e ideas. Bajo múltiples enfoques, las comunidades amazónicas continuamente revelan knowhow respecto de sus recursos, especialmente vegetales, con una alta riqueza de detalles, en un proceso ágrafo, surgido de la herencia sociocultural.

\section{El perfil sociodemográfico de las comunidades}

De manera acelerada, diversas comunidades establecieron viviendas a lo largo de ríos y carreteras, en una continua expansión urbano-rural. Silva et al. (2014) afirmaron que dicho crecimiento, además de generar cambios en los modos de vida de las personas y en las características del entorno biofísico ruralurbano-rural, interfiere en la forma de construir los espacios, tornando cada vez más difícil trazar los límites entre las zonas rurales y urbanas.

En cuanto a la realidad escolar, en la mayoría de los municipios y comunidades, este aspecto todavía es demasiado ineficiente, incluso, a pesar de los esfuerzos del gobierno para fomentar la alfabetización. Los etnógrafos generalmente encuentran interlocutores con un bajo nivel de escolaridad, con educación primaria incompleta o analfabetos/sin escolaridad (SANTOS et al., 2014; DAADY et al., 2016). Se evidenció que, en muchos de los lugares visitados, o no hay escuelas en los alrededores de las viviendas, debido a la distribución aislada de las poblaciones tradicionales amazónicas, o estas ofrecen solo el nivel primario de educación (VASQUEZ et al., 2014). La falta de oportunidades y el estímulo de la familia es otro agravante, ya que la escolaridad de algunos miembros de las comunidades es facilitada cuando se involucran con líderes comunitarios y buscan formación educativa como instrumento para luchar por sus derechos (FREITAS et al., 2013).

El saneamiento básico en las comunidades es a menudo precario o inexistente y hay escasez de agua potable para la satisfacción de las necesidades básicas de las familias (SOUSA, 2009). Para los que viven a orillas de los ríos, el suministro es directamente de él; para otros, el pozo es la principal fuente de agua. En la Reserva Extractiva Marina de Soure, Pará, el suministro de agua potable es deficiente, lo que obliga a los residentes a utilizar agua subterránea de pozos, artesianos o superficiales, como alternativa de captación. Estos pozos son instalados de manera precaria dejando el recurso hídrico altamente vulnerable a ser contaminado (LOBATO et al., 2014). Estos autores encontraron en las visitas in situ que las poblaciones de Caju-Úna y Povoado do Céu reciben agua solo dos veces por semana, a través de camiones cisterna 
proporcionados por el Municipio de Soure.

La basura, que en raras ocasiones se recoge, recibe la misma destinación que en tiempos pasados: se quema o se entierra en los patios traseros o se desecha al aire libre (MACIEL, 2012). Además, las excretas humanas no reciben tratamiento adecuado, lo que favorece al agravamiento constante de las enfermedades transmitidas por el agua, como las relacionadas con el sistema gastrointestinal y las enfermedades de la piel, registradas de forma rutinaria en diversos estudios (SOUSA, 2009; GIATTI et al., 2012; GOIS et al., 2016).

Pocas localidades reciben visitas regulares de médicos y/o enfermeros, o reciben medicamentos (GAMA et al., 2018). La tarea de monitorear el estado de salud de las personas se da, en la mayoría de los casos, a través de Agentes Comunitarios de Salud (ACSs), quienes, además de realizar procedimientos técnicos (vendajes, suturas y otros), suelen auxiliar con los remedios más urgentes y, también, prescribir medicinas tradicionales con plantas. Estas realidades evidenciadas mostraron una participación muy incipiente de los investigadores y de la gobernanza local en el suministro de medicamentos y orientaciones para mejorar la vida de las poblaciones estudiadas. Este panorama ha contribuido al aumento de casos de determinadas enfermedades, como la sífilis, el sida, la rabia, la enfermedad de Chagas; además de la permanencia crónica de diabetes, hipertensión arterial, diarrea, verminosis y enfermedades de la piel. Otros problemas sociales relacionados con el embarazo precoz, el alcoholismo y las drogas ilícitas han sido identificados como preocupaciones importantes en las zonas rurales. El caso es que el crecimiento poblacional avanza de manera no planificada (en las últimas dos décadas fue $41 \%$; superior al índice nacional de $31 \%$ ), y trae consigo graves consecuencias que contribuyen a la persistencia de la pobreza (REIS et al., 2011; STAEVIE, 2015).

\section{La etnobotánica en las ferias y mercados}

Las observaciones etnobotánicas que se realizan en ferias abiertas y mercados revelan el carácter indisociable existe entre los seres humanos con las plantas y remedios comercializados en estos lugares, que sirven como canales para mantener comportamientos que operan en religiones y religiosidades, remedios caseros y creencias. Entramados de saberes sobre las plantas que curan y purifican son construidos a través del comercio y el etnoconocimiento de los comerciantes y consumidores.

Las plantas para rituales en ferias y mercados nutren la medicina popular con un diverso arsenal de plantas, que curan y permiten la realización de los ritos religiosos. En los relatos de quienes buscan estas plantas y productos, hay una fuerte reverencia por las deidades, como caboclos, maestros, guías; además del uso destinado a atraer, amansar, mejorar la vida, etc. Carmo et al. (2015), en la feria 25 de Setiembre, Belém, Pará, identificaron 21 etnoespecies exclusivas para uso ritual que, según los expertos de la feria, sólo se realizan en su "efecto" o "resultado final" a través de ritos y creencias. En estos lugares, la búsqueda por estas prácticas curativas es realizada tanto por líderes evangélicos, practicantes del catolicismo, bendecidos y seguidores de religiones afrodescendientes, como por consumidores no especializados (HIEDA et al., 2011).

El ambiente de la feria, con todos los 'remedios' naturales permite que las prácticas terapéuticas sigan existiendo, aunque sea bajo una nueva perspectiva, una forma adaptada a los nuevos tiempos para 
obtener el remedio. Incluso si la planta no está en su patio, existe el valor del respeto por el uso exitoso, por el recuerdo de cuántas personas fueron tratadas y por eso, se generan nuevas expectativas seguras en la cura. En este contexto, las plantas son obtenidas por otros medios, de otras formas, para que el remedio continúe con la misma formulación.

El hábito de comprar las plantas en ferias y herboristerías forma parte de la rutina de D. Eliana y hoy en día se extiende a otras especialistas, que prescriben listas de plantas e indican su compra en las tiendas. Por otro lado, la falta de plantas en los terrenos puede ser un factor determinante en el éxito del tratamiento, ya que, dependiendo de la gravedad del caso, es importante comenzar lo antes posible. En este nuevo escenario, el tiempo de espera puede ser prolongado, además de la recepción, el diagnóstico, la formulación del listado de plantas a adquirir; la llegada de estas a manos de especialistas; la preparación del medicamento y el inicio del uso del medicamento.

Bitencourt et al. (2014) describieron la presencia de la biodiversidad en la vida cotidiana de los diferentes actores sociales que actúan en la feria en Guamá, Belém, Pará, enfatizando que esta diversidad biológica, transformada en el tiempo y el espacio, refleja la riqueza del medio ambiente amazónico en su naturaleza profunda y diversa, y en su aspecto cultural. Sobre esto, sin embargo, es necesario que los investigadores que desarrollan investigaciones en esta área sepan que los interlocutores pueden tener o no experiencia como herbolarios (conocedores experimentados de plantas) y, por lo tanto, el delineamiento del trabajo debe considerar el origen del conocimiento y la honestidad de los vendedores.

La cantidad de especies vegetales y productos que se comercializan en estos lugares tiene diferentes orígenes - huertos familiares, viveros, áreas forestales - que circulan hacia las áreas comerciales de los centros urbanos. En estos destinos se formulan recetas y medicamentos, con o sin estandarización - composición, empaque, recomendaciones de uso, almacenamiento, conservación, etc. Carmo et al. (2015) revelaron que, en el empaque de los 152 productos a base de plantas comercializadas en la Feria de 25 de Setiembre, en Belém, Pará, solo 63 tenían referencias sobre su composición y procedencia. Referencias sobre la indicación de uso, dosis y efectos tóxicos a menudo no se incluyen en las etiquetas o existen de manera ineficaz como orientación para el consumidor.

En las ferias, las muestras de hojas, flores, ramas y cortezas, comercializadas in natura o envasadas, pueden representar riesgos para la salud de los consumidores, por presentar estándares de calidad insuficientes para su comercialización. Algunos factores contribuyen a mantener esta situación, como: (1) la poca exigencia de los consumidores en cuanto a la calidad del producto, (2) falta de compromiso de algunos comerciantes que apuntan prioritariamente al lucro y no garantizan la calidad de sus productos; y la incipiente acción de fiscalización oficial, en gran parte debido al carácter informal de la actividad (LIMA et al., 2011). De esta forma, la eficacia terapéutica no es garantizada, ya que la población termina consumiendo materiales contaminados por impurezas (tierra, arena, excremento de animales, otras especies vegetales, coliformes fecales, etc.) y/o sin sus propiedades terapéuticas, debido a las malas condiciones de transporte, conservación y almacenamiento.

En estas circunstancias, se recomienda que, al investigar saberes tradicionales en ferias y mercados, 
el investigador seleccione sus interlocutores de forma bastante criteriosa y responsable. Comparar relatos entre sí y con literatura especializada; visitar los lugares de origen del cultivo de plantas y productos, así como conversar/entrevistar a sus fabricantes; y conocer antiguos consumidores que dan fe de la eficacia de los productos, son técnicas posibles para garantizar que la información transmitida se base realmente en conocimientos tradicionales ancestrales y no en el poder persuasivo de los comerciantes.

\section{Críticas, contribuciones y reflexiones sobre los estudios etnobotánicos}

La atención a la salud alimentaria de los pueblos y comunidades constituye un tema ausente. La lista de especies alimentarias, a pesar de mostrar nutrientes esenciales para el cuerpo humano, resulta insuficiente por ser demasiado restrictiva para las demandas del consumo diario. Viviendo básicamente de pescado, asaí y yuca, muchas comunidades tienen pocas opciones para el cultivo de verduras y hortalizas, dependiendo además de la estacionalidad de las especies frutales. Esto acarrea la presión sobre la fauna y la flora local, que también se ve severamente afectada por el avance de la deforestación. A esto se suma el aumento demográfico de estas familias, lo que genera desequilibrios no solo en términos alimentarios, sino además sociales (FURTADO et al., 2012). El monitoreo de la situación nutricional de las comunidades tradicionales debe investigarse como principal causa, ya que sus fuentes de alimentos no siempre están disponibles en la naturaleza.

Si bien el uso de la nomenclatura científica en los estudios etnobotánicos fue establecido como obligatorio, se han notado en los artículos otros problemas de identificación de plantas, tales como: (1) identificación científica realizada únicamente por el nombre vernáculo o popular, que está sujeto a regionalismos, originando un confusión con plantas tóxicas o plantas con ingredientes activos diferentes (por ejemplo, tres especies diferentes son conocidas popularmente como 'toronjil': C. citratus Stapf, Melissa officinalis L. y L. alba (Mill.) N.E.Br.); (2) falta de recolección de muestras botánicas y posterior incorporación en un herbario, ya que el uso de catálogos, imágenes o simplemente bancos virtuales es considerado un procedimiento dudoso para la nominación científica y, por lo tanto, debe ser evitado. Se sugiere que los materiales botánicos sean evaluados por taxonomistas o parataxonomistas y validados con número de registro de una colección institucionalizada.

Los resultados de los estudios etnobotánicos han apuntado debilidades en el logro de objetivos compartidos y de disfrute común para favorecer propuestas colectivas. Los esfuerzos intelectuales de los investigadores aún no alcanzan niveles más amplios en contextos pedagógicos, jurídicos y culturales para que puedan ser aplicados como dispositivos legales, formales e institucionalizados, respetando lo esencial para la reproducción física y social de las poblaciones. Homma (2013) abordó este tema enfatizando que a pesar de los grandes avances obtenidos en la comunidad científica local y nacional, aún no se ha podido inducir la gran transformación que la región está necesitando para resolver los problemas sociales, económicos y ambientales.

Se le da mucho énfasis a la biodiversidad vegetal, que es presentada en largas listas, generalmente valorada como el elemento investigativo prioritario y cuantitativo, dándose poco mérito a la comprensión de 
la supervivencia cultural de la población regional, que depende de productos tradicionales (nativos y exóticos), con potencial para generar ingresos y mejorar la calidad de vida. Ilustrando esta tesis, Dória (2014) explicó que la utilidad es el concepto central de la relación ser humano-naturaleza, y como tal, es histórica y no solo biológica, ya que las características biológicas solo tienen singularidad expresiva cuando se insertan en la vida social. Como resultado, las innumerables especies publicadas en los artículos solo adquieren "vida propia" a partir de la posición que ocupan en los procesos sociales que las comprenden.

La simple descripción y repetición de plantas en largas listas de identificación ha dificultado el avance de la etnobotánica como un área científica orientada a la resolución de problemas y con una reflexión funcional de lo descrito. Albuquerque et al. (2010) problematizaron que tales prácticas académicas no contribuyen a la solución de cuestiones básicas, como el entendimiento de los criterios que llevan a ciertos pueblos a seleccionar recursos biológicos, en ambientes específicos. También, debe haber una preocupación por discernir problemas similares en diferentes poblaciones tradicionales, respetando sus identidades propias. Tal limitación es visualizada en estudios que describen las plantas medicinales en la región amazónica como la principal forma de tratamiento de enfermedades, o el primer recurso adoptado. Información de este tipo, sin la debida justificación, desconoce el aspecto sociocultural de la población estudiada u omite problemas relacionados con el acceso o la precariedad de los servicios de salud formales.

Las investigaciones científicas de carácter etnobotánico pueden agregar otras áreas de conocimiento que se complementan como dispositivos para implementar acciones que permitan mejoras locales. Iniciativas de esta naturaleza fueron registradas por Lima et al. (2013) con los usos de la chontilla (Astrocaryum vulgare Mart.) y su aprovechamiento en la zona rural del municipio de Irituia, en el estado de Pará. Aprovechando el valor de esta palma como parte de la cultura tradicional de agricultores familiares, ribereños y quilombolas, la Universidad Federal de Pará (UFPA) en colaboración con el Municipio de Irituia, estudiaron la instalación de una planta de procesamiento de oleaginosas en el municipio, que incluye la chontilla como potencial materia prima. Esta propuesta estimuló la producción en sistemas agroforestales, reemplazando la agricultura de tala y quema, y conciliando la conservación ambiental con el Desarrollo Rural Territorial.

El Convenio sobre Diversidad Biológica provocó debates sobre la distribución de los beneficios resultantes del uso de recursos. Entendiendo la importancia de esto para la conservación de la biodiversidad, se viene recomendando que la investigación realizada sea devuelta a la comunidad. Quinteiro et al. (2014) comentaron que el análisis de artículos científicos en etnobotánica muestra casos específicos de retorno y aplicabilidad de los estudios, y también, que poco se escucha sobre las necesidades de las comunidades locales, o sobre propuestas alternativas para resolver problemas existentes o explorar otras formas cognitivas inherentes al uso de recursos vegetales.

Como resultado de la investigación socioambiental realizada en patios traseros periurbanos del barrio Mutirão, Abaetetuba, Pará (LOBATO et al., 2017), el retorno para la comunidad fue pensado, construido y desarrollado de manera dialéctica entre los actores involucrados en la investigación, culminando con la elaboración de diseños temáticos referentes a los patios y la ejecución de un taller de reciclaje. Estas 
acciones resultaron en planes futuros con ilustraciones de flora y fauna, así como la satisfacción e interés de cada uno de los participantes por conocer las técnicas de elaboración de objetos y su posible comercialización. En la comunidad ribereña de Río Urubueua, Abaetetuba, Pará, las enseñanzas de las mujeres más experimentadas en la preparación de tés medicinales fueron socializadas y organizadas en formato de folletos, como forma de retribución al público objetivo. Fueron presentadas las plantas, métodos de preparación, dosis e indicaciones etnofarmacológicas (MOURA et al., 2016).

\section{CONCLUSIONES}

Corresponde a la ciencia no solo identificar los problemas que enfrentan las comunidades, especialmente en lo referente al acceso a tecnologías o capacitación para el manejo y productividad de los recursos naturales, sino que también urge realizar una nueva lectura de todos los conocimientos ya producidos, y que aguardan medidas más transformadoras y no solo colaborativas, es decir, asignar funciones prácticas de los proyectos que vayan más allá del academicismo teórico.

Es importante que los trabajos etnográficos incentiven la movilización de las comunidades para luchar por sus derechos y su empoderamiento social, legal y económico. Esto solo ocurrirá, de hecho, si las investigaciones desarrolladas sirven como documentos para resolver los problemas relacionados con el mantenimiento del territorio, a fin de asegurar y sustentar las formas de vida de las poblaciones estudiadas. Se hace imprescindible trabajar en diagnósticos participativos específicos para cada grupo social, enfocándose en la elaboración de mapeos sociales, que pueden convertirse en instrumentos de gestión, de territorialidad (falta de regularización de títulos de propiedad), que sirvan para el combate a la deforestación, la prohibición del turismo desordenado en las zonas prioritarias y para la toma de medidas preventivas ante la pérdida de la reproducción social de las poblaciones y alteraciones en sus formas de vida.

En este contexto, diversas estrategias pueden ser articuladas y desarrolladas dentro de cada comunidad, respetando una construcción mutua, arraigada en un contexto particular. Entre estas: ofrecer mano de obra calificada para la capacitación e implementación de sistemas de uso del suelo y optimización del agua natural; implementar la atención de salud; educar con y en el medio ambiente; así como desarrollar acciones dirigidas a la construcción de huertos comunitarios; instrucción sobre el potencial fitoterapéutico de productos de la biodiversidad local; valorar las identidades locales y respetar las diferencias en las relaciones laborales entre géneros; mediación con agencias públicas para la implementación de escuelas y logística para el transporte estudiantil; entre otras que representen debates importantes para el presente y futuro de los pueblos tradicionales. Tales intervenciones pueden contribuir a: (1) la construcción de individuos más críticos y poblaciones más autónomas; (2) la comprensión de la naturaleza como una interacción sistémica de todos los procesos vivos y no vivos; (3) una participación efectiva en el proceso de conservación de la biodiversidad; y (4) el mantenimiento de las formas de vida tradicionales de las comunidades. 


\section{REFERENCIAS}

ALBUQUERQUE, M. B. B; FARO, M. C. S.. Saberes de cura: um estudo sobre pajelança cabocla e mulheres pajés da Amazônia. Rev. Bras. de Hist. das Relig., v.5, n.13, p.57-72, 2015. DOI: https://doi.org/10.4025/rbhranpuh.v5i13.30252

ALBUQUERQUE, U. P.; LUCENA, R. F. P.; CUNHA, L. V. F. C.. Métodos e Técnicas na Pesquisa Etnobiológica e Etnoecológica. Pernambuco: NUPPEA, 2010.

ALVES, K. N. L.; MESQUITA, U. O.; LEÃO, V. M.; GOIS, M. A. F.; VIEIRA, E. F. M.; NEVES, R. H. P.; SOUZA, J. P. S.; LUCAS, F. C. A.; COSTA, J. C. M.. Centros depositórios de plantas medicinais: herbários como instrumento de gestão da biodiversidade. Revista Fitos, v.11, n.1, p.26-37, 2017. DOI: https://doi.org/10.5935/2446-4775.20170012

ASTOLFI FILHO, S.; SILVA, C. G. N.; BIGI, M. F. M. A.. Bioprospecção e biotecnologia. Parcerias Estratégicas, v.19, n.38, p.45-80, 2015.

BITENCOURT, B. L. G.; LIMA, P. G. C.; BARROS, F. B. Comércio e uso de plantas e animais de importância mágicoreligiosa e medicinal no Mercado Público do Guamá, Belém do Pará. Revista Faculdade Santo Agostinho, v.11, n.3, p.96158, 2014.

BOLETIM INFORMATIVO. Território de Povos e Comunidades Tradicionais no Arquipélago de Marajó. Nova Cartografia Social da Amazônia, 2014.

BRASIL. Decreto n.6040, de 7 de fevereiro de 2007. Institui a Política Nacional de Desenvolvimento Sustentável dos Povos e Comunidades Tradicionais. Brasília: DOU, 2007.

CARMO, T. N.; LUCAS, F. C. A.; LOBATO, G. J. M.; GURGEL, E. S. C... Plantas Medicinais e Ritualísticas comercializadas na Feira da 25 de Setembro, Belém, Pará. Enciclopédia Biosfera, v.11, n.21, p.3440-3467, 2015.

CARNEIRO, D. B.; BARBOZA, M. S. L.; MENEZES, M. P.. Plantas nativas úteis na Vila dos Pescadores da Reserva Extrativista Marinha Caeté-Taperaçu, Pará, Brasil. Acta Bot. Bras., v.24, n.4, p.1027-1033, 2010. DOI: https://doi.org/10.1590/S0102-33062010000400017

CASSINO, M. F.. Estudo etnobotânico de plantas medicinais em comunidades de várzea do rio Solimões, Amazonas e aspectos farmacognósticos de Justicia pectoralis Jacq. mutuquinha (Acanthaceae). Dissertação (Mestrado em Botânica) - Universidade Federal do Amazonas, Manaus, 2010.

COSTA, G. S.. Desenvolvimento rural sustentável com base no paradigma da agroecologia. Belém: Universidade Federal do Pará, 2006.

DAADDY, M. D. V.; SANTOS, C.; BRANDÃO, R. M. L.; AMANAJÁS, R. D.; RIBEIRO, A. B. N.. Pesca do apaiari, Astronotus ocellatus (Agassiz, 1831), e perfil socioeconômico dos pescadores artesanais de uma região da Amazônia brasileira. Boletim do Museu Paraense Emílio Goeldi. Ciências Humanas, v.11, n.2, p.363-378, 2016. DOI: https://doi.org/10.1590/1981.81222016000200002

DAS, T.; DAS, A. K.. Conservation of Plant Diversity in Rural
Homegardens with Cultural and Geographical Variation in Three Districts of Barak Valley, Northeast India. In: Economic Botany, v.69, n.1 p.57-71, 2015. DOI: http://doi.org/10.1007/s12231-015-9299-6

DÓRIA, C. A.. Ambiente e Sociedade na Amazônia: uma análise dos cronistas-naturalistas. Garamond: Rio de Janeiro, 2014.

FAINGUELERNT, M. B.. Belo Monte: o Estado democrático de direito em questão. Rio de Janeiro: Apicuri, 2013.

Freitas, J. L.; SANTOS, E. S.; SILVA, R. B. L.; SILVA, T. L.. Comparação e análise de sistemas de uso da terra de agricultores familiares na Amazônia. Biota Amazonia, v.3, n.1, p.100-108, 2013. DOI: http://dx.doi.org/10.18561/21795746/biotaamazonia.v3n1p100-108

FURTADO, L. G.; SILVEIRA, I. M.; SANTANA, G.. Reserva Extrativista Marinha Mãe Grande, Curuça, Pará, Brasil: estudo etnoecológico e sociocultural. Belém: Museu Paraense Emílio Goeldi, 2012.

GAMA, A. S. M.; FERNANDES, T. G.; PARENTE, R. C. P.; SECOLI, S. R.. Inquérito de saúde em comunidades ribeirinhas do Amazonas, Brasil. Cadernos Saúde Pública, v.34, n.2, 2018. DOI: https://doi.org/10.1590/0102$\underline{311 \times 00002817}$

GANDOLFO, E. S.; HANAZAKI, N.. Etnobotânica e urbanização: conhecimento e utilização de plantas de restinga pela comunidade nativa do distrito do Campeche (Florianópolis, SC). Acta Botanica Brasilica, v.25, n.1, p.168177, 2011. DOI: https://doi.org/10.1590/S0102$\underline{33062011000100020}$

GERMANO, C. M.; LUCAS, F. C.; TAVARES-MARTINS, A. C. C.; MOURA, P. H. B.; LOBATO, G. J. M.. A Comunidades ribeirinhas e palmeiras no município de Abaetetuba, Pará, Brasil. Scientia Plena, v.10, n.11, p.1-10, 2014.

GIATTI, L. L.; CUTOLO, A. S.. Acesso à água para consumo humano e aspectos de saúde pública na Amazônia Legal. Ambiente \& Sociedade, v.15, n.1, p.93-109, 2012. DOI: http://dx.doi.org/10.1590/S1414-753X2012000100007

GOIS M. A. F.; LUCAS, F. C. A.; COSTA, J. C. M.; MOURA, P. H. B.; LOBATO, G. J. M.. Etnobotânica de espécies vegetais medicinais no tratamento de transtornos do sistema gastrointestinal. Rev. Bras. de Plant. Med., v.18, n.2, p.547557, 2016. DOI: http://doi.org/10.1590/1983-084X/15 170

HIEDA, M. F.; ALVES, A. A.. Intolerância religiosa a umbanda: a perseguição da Igreja Universal do Reino de Deus aos umbandistas. Rev. Bras. de Hist. das Relig., v.3, n.9, p.19832859, 2011.

HOMMA, A. K. O.. Amazônia: os avanços e os desafios da pesquisa agrícola. Parcerias Estratégicas, v.18, n.36, p.33-54, 2013.

LIMA, L. P.. Ocorrências e usos do tucumã (Astrocaryum vulgare Mart.) em comunidades ribeirinhas, quilombolas e de agricultores tradicionais no município de Irituia, Pará. Amazônica: Revista de Antropologia, v.5, n.3, p.762-778, 
2013. DOI: http://hdl.handle.net/11449/137513

LIMA, P. G. C.; COELHO-FERREIRA, M. R.; OLIVEIRA, R.. Plantas medicinais em feiras e mercados públicos do Distrito Florestal Sustentável da BR-163, estado do Pará, Brasil. Acta Botânica Brasílica, v.25, n.2, p.422-434, 2001. DOI: https://doi.org/10.1590/S0102-33062011000200018

LIRA, T. M.; CHAVES, M. P. S. R.. Comunidades ribeirinhas na Amazonia: organização sociocultural e política. Interações, Campo Grande, v.17, n.1, 2016. DOI:

http://dx.doi.org/10.20435/1518-70122016107

LOBATO, G. J. M.; LUCAS, F. C. A.; GURGEL, E. S. C.. Composição nutricional de espécies frutíferas em quintais urbanos, Abaetetuba, Pará, Brasil. Cadernos Agroecológicos, v.10, n.3, p.1-6, 2016.

LOBATO, G. J. M.; LUCAS, F. C. A.; TAVARES-MARTINS, A. C. C.; JARDIM, M. A.; MARTORANO, L. G.. Diversidade de uso e aspectos socioambientais de quintais urbanos em Abaetetuba, Pará, Brasil. Revista Brasileira de Agroecologia, v.12, n.2, p.95-105, 2017.

LOBATO, G. J. M.; TAVARES-MARTINS, A. C. C.; LUCAS, F. C. A.; MORALES, G. P.; ROCHA, T. T.. Reserva Extrativista Marinha de Soure, Pará, Brasil: modo de vida das comunidades e ameaças ambientais. Biota Amazonia, v.4, n.4, p.66-74, 2014. DOI: http://dx.doi.org/10.18561/21795746/biotaamazonia.v4n4p66-74

MACIEL, R. C. G.; SOUZA, E. F.; ROSSETTO, A. C. F.; SOUZA, G E. A. B.; JUNIOR, V. A. G.. Produção de lixo na floresta: um novo olhar para as florestas acreanas. Revista de Estudos Sociais, v.14, n.27, p.28-44, 2012.

MARTINS, A. G.; ROSÁRIO, D. L.; BARROS, M. N.; JARDIM, M. A. G.. Levantamento etnobotânico de plantas medicinais, alimentares e tóxicas da Ilha do Combu, Município de Belém, Estado do Pará, Brasil. Rev. Bras. de Farm., v.86, n.1, p.2130, 2005.

MAUÉS, R. H.. O perspectivismo indígena é somente indígena? Cosmologia, religião, medicina e populações rurais na Amazônia. Mediações: Revista de Ciências Sociais, v.17, n.1, p.33-61, 2012.

MORANM E.. Adaptabilidade Humana: Uma Introdução à Antropologia Ecológica. 2 ed. São Paulo: Edusp - Editora da Universidade de São Paulo, 512 p.2010.

MOURA, P. H. B.; LUCAS, F. C. A.; TAVARES-MARTINS, A. C. C.; LOBATO, G. J. M.; GURGEL, E. S. C.. Etnobotânica de chás terapêuticos em Rio Urubueua de Fátima, Abaetetuba-Pará, Brasil. Biotemas, v.29, n.2, p.77-88, 2016. DOI: https://doi.org/10.5007/2175-7925.2016v29n2p77

OLIVEIRA F. C.; ALBUQUERQUE, U. P.; FONSECA-KRUEL, V. S.; HANAZAKI, N.. Avanços nas pesquisas etnobotânicas no Brasil. Acta Botanica Brasilica, v.23, n.2, p.590-605, 2009. DOI: http://dx.doi.org/10.1590/S0102-33062009000200031

OLIVEIRA, M. C. C.; ALMEIDA, J.; SILVA, L. M. S.. Diversificação dos sistemas produtivos familiares: reflexos sobre as relações sociedade-natureza na Amazônia Oriental. Novos Cadernos NAEA, v.14, n.2, p.61-88, 2011.
QUINTEIRO, M. M. C.; TAMASHIRO, A. M. G.; MORAES, M. G.. Formas de retorno da pesquisa etnobotânica à comunidade no paradigma da complexidade ambiental e Educação Ambiental. Revista Brasileira de Educação Ambiental, v.8, n.1, p.91-99, 2014

REIS, M. L.; LAPA, U. C; CHAVES, M. P. S. R.. Desigualdades sociais e a sustentabilidade na Amazônia. 2010.

ROBERT, P.; LÓPEZ, C.. Agriculturas amazônicas: cultivando plantas, saberes, paisagens e ideias. Boletim do Museu Paraense Emílio Goeldi. Ciências Humanas, v.7, n.2, p.289292, 2012.

SANTOS, A. C.; FREITAS, J. L.; SILVA, R. B. L.; MORAES, L. L. C.; FILHO, J. R. M.; JUNIOR, F. O. C.; CANTUÁRIA, P. C.

Caracterização da atividade extrativa vegetal na comunidade São Tomé, Ferreira Gomes, Amapá, Brasil. Biota Amazonia, v.5, n.4, p.42-47, 2015. DOI:

http://dx.doi.org/10.18561/2179-

5746/biotaamazonia.v5n4p42-47

SANTOS, M. N.; CUNHA, H. F. A.; LIRA-GUEDES, A. C.; GOMES, S. C. P.; GUEDES, M. C.. Saberes tradicionais em uma unidade de conservação localizada em ambiente periurbano de várzea: etnobiologia da andirobeira (Carapa guianensis Aublet). Boletim do Museu Paraense Emílio Goeldi. Ciências Humanas, v.9, n.1, p.93-108, 2014.

SANTOS, M. R. A.; LIMA, M. R.; FERREIRA, M. G. R.. Uso de plantas medicinais pela população de Ariquemes, em Rondônia. Horticultura Brasileira, v.26, n.2, p.244-250, 2008.

SANTOS, M. R. A.; LIMA, M. R.; FERREIRA, M. G. R.; OLIVEIRA, C. L. L. G.. Medicinal plants used in Rondônia, Western Amazon, Brazil. Revista Brasileira de Plantas Medicinais, v.16, n.3, p.707-720, 2014. DOI: http://dx.doi.org/10.1590/1983-084x/13 102

SANTOS, R. S.; COELHO-FERREIRA, M. R.. Estudo etnobotânico de Mauritia flexuosa L. f. (Arecaceae) em comunidades ribeirinhas do Município de Abaetetuba, Pará, Brasil. Acta Amazonica, v.42, n.1, p.1-10, 2012. DOI: https://doi.org/10.1590/S0044-59672012000100001

SEMEDO, R. J. C. G.; BARBOSA, R. I.. Árvores frutíferas nos quintais urbanos de Boa Vista, Roraima, Amazônia brasileira. Acta Amazonica, v.37, n.4, p.497-504, 2007. DOI: http://dx.doi.org/10.1590/50044-59672007000400003

SILVA, E. R. R; SABLAYROLLES, M. G. P.. Caracterização das Atividades de Agricultura Urbana: Um Estudo de Caso em Santarém-Pará. Cadernos de Agroecologia, v.9, n.4, p.1-13, 2014.

SILVA, P. H.; BARROS, M. S.; OLIVEIRA, Y. R.; ABREU, M. C.. A etnobotânica e as plantas medicinais sob a perspectiva da valorização do conhecimento tradicional e da conservação ambiental. Revista Brasileira de Ciências Ambientais, v.9, n.2, p.67-86, 2015. DOI: http://dx.doi.org/10.18316/1981$\underline{8858.12}$

SOUSA, I. S.. As condições de vida e saneamento nas comunidades da área de influência do gasoduto CoariManaus em Manacapuru, AM. Hygeia, v.5, n.9, p.88-98, 2009. 
STAEVIE, P. M.. Crescimento demográfico e exclusão social nas capitais periféricas da Amazônia. Textos e Debates, v.1, n.28, p.185-204, 2015.

TOLEDO, V. M.; BARRERA-BASSOLS, N. B.. A etnoecologia: uma ciência pós-normal que estuda as sabedorias tradicionais. Desenvolvimento e Meio Ambiente, n.20, p.3145, 2010.

VAL, A. L.. Amazônia um bioma multinacional. Ciência e Cultura, v.66, n.3, p.20-24, 2014.

VASQUEZ, S. P. F.; MENDONCA, M. S.; NODA, S. N.. Etnobotânica de plantas medicinais em comunidades ribeirinhas do Município de Manacapuru, Amazonas, Brasil. Acta Amazônica, v.44, n.4, p.457-472, 2014.

VEIGA, J. B.; SCUDELLER, V. V.. Etnobotânica e medicina popular no tratamento de malária e males associados na comunidade ribeirinha Julião - baixo Rio Negro (Amazônia Central). Revista Brasileira de Plantas Medicinais, v.17, n.4, p.737-747, 2015. DOI: http://doi.org/10.1590/1983$\underline{084 X / 14039}$

VENDRUSCOLO, G. S.; MENTZ, L. A.. Uso de plantas medicinais por uma comunidade rural de Porto Alegre, Rio Grande do Sul, Brasil. In: SILVA, V. A.; ALMEIDA, A. L. S.; ALBUQUERQUE, U. P.. Etnobiologia e Etnoecologia: pessoas \& natureza na América Latina. Recife: NUPEEA, 2010. p.211227.

VIEIRA, I. C. G.; TOLEDO, P. M.; SANTOS JÚNIOR, R. A.. O Ambiente e sociedade na Amazônia: uma abordagem interdisciplinar. Garamond, Rio de Janeiro, 498p.2014.

VIEIRA F. P.; SOUZA, L. B.. A Educação Ambiental com as comunidades tradicionais: outras trajetórias de sustentabilidades. Notandum, v.21, n.47, p.153-173, 2018. DOI: https://doi.org/10.4025/notandum.47.10

WAWZYNIAK, J. V.. "Engerar" uma categoria cosmológica sobre pessoa, saúde e corpo. Ilha: Revista de Antropologia, v.5, n.2, p.33-55, 2003.

A CBPC - Companhia Brasileira de Produção Científica (CNPJ: 11.221.422/0001-03) detém os direitos materiais desta publicação. Os direitos referem-se à publicação do trabalho em qualquer parte do mundo, incluindo os direitos às renovaç̃oses, expansões e disseminações da contribuiç̃o, bem como outros direitos subsidiários. Todos os trabalhos publicados eletronicamente poderão posteriormente ser publicados em coletâneas impressas sob coordenação da Sustenere Publishing, da Companhia Brasileira de Produção Científica e seus parceiros autorizados. Os (as) autores (as) preservam os direitos autorais, mas não têm permissão para a publicação da contribuição em outro meio, impresso ou digital, em português ou em tradução. 